\title{
Monitoring of Motor and Somatosensory Evoked Potentials During Spine Surgery: Intraoperative Changes and Postoperative Outcomes
}

\author{
Shin Hye Chang, $\mathrm{MD}^{1,2}$, Yoon Ghil Park, MD, $\mathrm{PhD}^{1,2}$, Dae Hyun Kim, MD ${ }^{3}$, Seo Yeon Yoon, $\mathrm{MD}^{1,2}$
}

${ }^{1}$ Department of Rehabilitation Medicine, Gangnam Severance Hospital, Seoul; ${ }^{2}$ Rehabilitation Institute of Neuromuscular Disease, Yonsei University College of Medicine, Seoul; ${ }^{3}$ Veteran Health Service Medical Center, Seoul, Korea

Objective To evaluate whether the combination of muscle motor evoked potentials (mMEPs) and somatosensory evoked potentials (SEPs) measured during spinal surgery can predict immediate and permanent postoperative motor deficits.

Methods mMEP and SEP was monitored in patients undergoing spinal surgery between November 2012 and July 2014. mMEPs were elicited by a train of transcranial electrical stimulation over the motor cortex and recorded from the upper/lower limbs. SEPs were recorded by stimulating the tibial and median nerves.

Results Combined mMEP/SEP recording was successfully achieved in 190 operations. In 117 of these, mMEPs and SEPs were stable and 73 showed significant changes. In 20 cases, motor deficits in the first 48 postoperative hours were observed and 6 patients manifested permanent neurological deficits. The two potentials were monitored in a number of spinal surgeries. For surgery on spinal deformities, the sensitivity and specificity of combined mMEP/ SEP monitoring were $100 \%$ and $92.4 \%$, respectively. In the case of spinal cord tumor surgeries, sensitivity was only $50 \%$ but SEP changes were observed preceding permanent motor deficits in some cases.

Conclusion Intraoperative monitoring is a useful tool in spinal surgery. For spinal deformity surgery, combined mMEP/SEP monitoring showed high sensitivity and specificity; in spinal tumor surgery, only SEP changes predicted permanent motor deficits. Therefore, mMEP, SEP, and joint monitoring may all be appropriate and beneficial for the intraoperative monitoring of spinal surgery.

Keywords Motor evoked potentials, Somatosensory evoked potentials, Intraoperative neurophysiological monitoring, Spine

Department of Rehabilitation Medicine, Gangnam Severance Hospital, 211 Eonju-ro, Gangnam-gu, Seoul 06273, Korea. Tel: +82-2-2019-3493, Fax: +82-2-3463-7585, E-mail: DRTLC@yuhs.ac

ORCID: Shin Hye Chang (http://orcid.org/0000-0003-1749-3940); Yoon Ghil Park (http://orcid.org/0000-0001-9054-5300); Dae Hyun Kim (http:// orcid.org/0000-0002-5065-4286); Seo Yeon Yoon (http://orcid.org/0000-0002-0365-2923).

@ This is an open-access article distributed under the terms of the Creative Commons Attribution Non-Commercial License (http://creativecommons.org/ licenses/by-nc/4.0) which permits unrestricted noncommercial use, distribution, and reproduction in any medium, provided the original work is properly cited. Copyright $\odot 2016$ by Korean Academy of Rehabilitation Medicine 


\section{INTRODUCTION}

Postoperative motor deficits following neurosurgical procedures result in significant morbidity and mortality rates as well as increased medical costs associated with the extended length of stay and rehabilitation [1]. For about a decade, intraoperative neurophysiological monitoring has been performed during spine and spinal cord surgery in an attempt to monitor, predict, and mitigate such negative outcomes [2].

For much of this time, only somatosensory evoked potentials (SEPs) were monitored during spinal cord procedures. However, SEPs do not reflect the functional integrity of motor pathways. The assumption that they do has resulted in a number of so called 'false negative' results, i.e., emergence of postoperative motor deficits despite unchanged intraoperative SEPs [3].

In recent years, muscle motor-evoked potentials (mMEPs) are also monitored. Many neurosurgeons and orthopedic surgeons now advocate mMEP monitoring for all spinal surgery, since they better predict good postoperative motor outcomes than the use of SEPs alone.

In addition to this predictive power, mMEP data recording benefits from a high temporal resolution; the data may be updated on the order of seconds providing the surgeon with 'real time' information regarding possible surgical trauma. Recent studies have reported the benefits of combining mMEP with SEP monitoring; such benefits have been clearly demonstrated during spinal cord surgery by taking into account the complementary information from two independent systems, these measurements provide increased sensitivity and a reduced risk of false negatives $[4,5]$.

Most reports, however, have focused on immediate postoperative motor deficits. Very few studies have follow-ups of any substantial length or reported whether postoperative deficits persisted or were resolved.

Therefore, this study aimed to compare intraoperative changes in mMEPs, SEPs, and both together with the neurological outcomes of spinal surgery, in order to demonstrate any possible advantages of methods using a combined over a single modality. In addition, the correlation between spinal surgery types and intraoperative changes was assessed in our institution.

\section{MATERIALS AND METHODS}

\section{Subjects}

Between November 2012 and July 2014, intraoperative mMEP and SEP monitoring was attempted during 190 spinal operations. All operations were performed by spinal surgeons with extensive experience in the type of spinal surgery conducted. Of the 190 patients, 90 were female. Patients ranged in age from 11 to 83 years (mean, 62.4 years). Prospectively analyzed patient data included medical records, intraoperative monitoring records, operative narratives, anesthesia records, and outpatient clinical notes. Preoperatively, 111 patients were neurologically intact and 79 patients had motor deficits. The baseline characteristics of all participants were listed in Table 1.

\section{Procedures}

mMEP and SEP monitoring was successful in all patients, who were then subdivided into three groups by diagnosis. The first group (Group 1) comprised 54 patients who underwent operations for spinal cord tumors. The second group (Group 2) was an equally sized group of patients with spinal deformities, and operations were performed in cases of scoliosis or kyphosis. The third group (Group 3), on which other spinal operations were performed, was composed of 82 patients diagnosed with disc herniation, ossification of the posterior longitudinal ligament (OPLL), spinal stenosis, and similar conditions. The study was approved by the ethics committee of the Gangnam Severance Hospital.

\section{Anesthesia}

A short-acting muscle relaxant (rocuronium) was used to facilitate tracheal intubation and ventilation; no paralytic agents were subsequently used. Anesthesia was administered intravenously using continuous application of propofol (6-8 $\mathrm{mg} / \mathrm{kg} / \mathrm{hr})$ and remifentanil (0.15-2 $\mu \mathrm{g} / \mathrm{kg} / \mathrm{min}$ ). Desflurane with nitrous oxide was used as a supplement in 7 surgeries. The level of neuromuscular blockage was assessed by posterior tibial nerve stimulation at the ankle with a train-of-four stimulus to observe muscle twitches.

Direct radial artery pressure, ECG, end-tidal carbon dioxide concentration, pulse oximetry, and temperature were monitored. All patients were kept normothermic 
Table 1. Summary of characteristics in 190 patients who underwent spinal surgery (group A, preoperatively motor intact; group B, preoperatively motor deficit)

\begin{tabular}{|c|c|c|c|c|}
\hline Characteristic & Total $(n=190)$ & Group A $(n=111)$ & Group B $(n=79)$ & p-value \\
\hline Age (yr) & $62.4(11-83)$ & $61.4(13-83)$ & $65.3(11-79)$ & 0.195 \\
\hline Sex & & & & 0.110 \\
\hline Female & $90(47.3)$ & $58(52.2)$ & $32(40.5)$ & \\
\hline Male & $100(52.6)$ & $53(47.7)$ & $47(59.4)$ & \\
\hline Diagnosis & & & & 0.347 \\
\hline \multicolumn{5}{|l|}{ Tumor (Group 1) } \\
\hline IDEM & $34(17.8)$ & $17(15.3)$ & $17(21.5)$ & \\
\hline IMSCT & $10(5.2)$ & $6(5.4)$ & $4(5.1)$ & \\
\hline ED & $6(3.2)$ & $3(2.7)$ & $3(3.8)$ & \\
\hline Syringomyelia & $4(2.1)$ & $4(3.6)$ & $0(0.0)$ & \\
\hline \multicolumn{5}{|l|}{ Spinal deformity (Group 2) } \\
\hline Idiopathic scoliosis & $30(15.7)$ & $30(27.0)$ & $0(0.0)$ & \\
\hline Congenital scoliosis & $2(1.0)$ & $1(0.9)$ & $1(1.3)$ & \\
\hline Neuromuscular scoliosis & $18(9.4)$ & $3(2.7)$ & $15(19.0)$ & \\
\hline Kyphosis & $4(2.1)$ & $2(1.8)$ & $2(2.5)$ & \\
\hline \multicolumn{5}{|c|}{ Miscellaneous disorders (Group 3) } \\
\hline Trauma & $5(2.6)$ & $4(3.6)$ & $1(1.3)$ & \\
\hline OPLL & $36(18.9)$ & $22(19.8)$ & $14(17.7)$ & \\
\hline Disc prolapse & $36(18.9)$ & $16(14.4)$ & $20(25.3)$ & \\
\hline Infection & $2(1.0)$ & $1(0.9)$ & $1(1.3)$ & \\
\hline Ankylosing spondylosis & $2(1.0)$ & $2(1.8)$ & $0(0.0)$ & \\
\hline Spondylolisthesis & $1(0.5)$ & $0(0.0)$ & $1(1.3)$ & \\
\hline
\end{tabular}

Values are presented as mean (range) or number (\%).

Group 1, spinal cord tumor operations; Group 2, spinal deformity operations; Group 3, other spinal operations; IDEM, intradural extramedullary tumors; IMSCT, intramedullary spinal cord tumors; ED, extradural tumors; OPLL, ossification of the posterior longitudinal ligament.

with a warming blanket. Normotensive anesthesia was maintained throughout the operation.

\section{Technique for SEP monitoring}

Somatosensory evoked potentials (Cascade; Cadwell Industries Inc., Kennewick, WA, USA) were elicited by stimulation of the median nerve at the wrist and the posterior tibial nerve at the ankle (intensity $20 \mathrm{~mA}$, duration $0.2 \mathrm{~ms}$, with a repetition rate of $5 \mathrm{~Hz}$ ). Recordings were performed via surface electrodes from the scalp at C3 (right median nerve stimulation), C4 (left median nerve stimulation), and $\mathrm{Cz}$ (right and left tibial nerve stimulation) and from a reference electrode at $\mathrm{FPz}$ according to the international 10-20 EEG system.

\section{Technique for mMEP monitoring}

Multipulse transcranial electrical stimulation was performed using a commercially available Cascade IOM electrical stimulator (Cadwell Industries Inc.). Transcranial electric motor-evoked potentials were recorded bilaterally from the biceps brachii and abductor pollicis brevis muscles in the upper extremities, and bilaterally from the tibialis anterior and abductor hallucis muscles in the lower extremities using a pair of needle electrodes inserted $3 \mathrm{~cm}$ apart in each muscle. Short trains of 5-7 square-wave stimuli of $0.5 \mathrm{~ms}$ duration and an interstimulus interval (ISI) of $3 \mathrm{~ms}$ were delivered at a repetition rate of up to $2 \mathrm{~Hz}$ through needle electrodes placed at $\mathrm{C} 1$ and $\mathrm{C} 2$ scalp sites. A C1/C2 montage is preferentially used to elicit right extremity mMEPs, while $\mathrm{C} 2 / \mathrm{C} 1$ is preferable for left extremity mMEPs. All electrode sites were 
in accordance with the international 10-20 system.

The stimulation intensity was gradually increased (50 $\mathrm{V}$ increments from $250 \mathrm{~V}$ to a maximum of $400 \mathrm{~V}$ ) until mMEP amplitudes were maximized above a minimum of $10 \mathrm{mV}$.

\section{Electrophysiological monitoring}

Electrophysiological monitoring was performed throughout the surgical procedures in our series. Baseline readings were obtained after exposure of the operative zone. Waveforms were analyzed for their peak-topeak amplitude.

A reduction of more than $50 \%$ in, mMEP baseline amplitude elicited by direct cortical stimulation was considered a significant intraoperative change indicative of impairment of functional integrity of the motor pathway. An SEP latency increase of more than $10 \%$ from baseline was regarded as significant. If significant EP changes occurred, the surgeons were informed and asked to stop the current surgical intervention. If the EP wave returned to normal, operative activities were resumed. If there was no signal reversal, even after reversing the surgical course and removing any implants, cessation of the procedure was considered.

\section{Neurological examination}

The motor status of each patient was evaluated before surgery, within 48 hours postoperatively, and 4 weeks later. Neurological condition was calculated on the Medical Research Council (MRC) scale for muscle strength (with the 10 key muscles of the International Standards for Neurological Classification of Spinal Cord Injury [ISNCSCI] motor score). The strength of these 10 key muscles ranged from 0 to 5 . Thus, the total score ranged from 0 to

Table 2. Patients with permanent motor deficits in spinal operations

\begin{tabular}{|c|c|c|c|c|c|}
\hline Case no. & Age/sex & Diagnosis & Level of lesion & EP changes & Motor deficit \\
\hline 1 & $51 / \mathrm{F}$ & Epidermoid cyst & L2-L3 & Both MEP and SEP change & Permanent \\
\hline 2 & $75 / \mathrm{F}$ & Glioblastoma & $\mathrm{C} 3-\mathrm{C} 4$ & SEP change & Permanent \\
\hline 3 & $24 / \mathrm{M}$ & Anaplastic astrocytoma & $\mathrm{C} 2-\mathrm{T} 1$ & SEP change & Permanent \\
\hline 4 & $30 / \mathrm{F}$ & $\begin{array}{l}\text { Spinal cord tumor, } \\
\text { uncertain type }\end{array}$ & $\mathrm{C} 2-\mathrm{C} 4$ & Both MEP and SEP change & Permanent \\
\hline 5 & $69 / \mathrm{M}$ & Cervical myelopathy, OPLL & C3-C5 & Both MEP and SEP change & Permanent \\
\hline 6 & $81 / \mathrm{M}$ & Cervical myelopathy, OPLL & C3-C5 & Both MEP and SEP change & Permanent \\
\hline 7 & $43 / \mathrm{F}$ & T3 chance fracture & T3-T5 & Both MEP and SEP change & Transient \\
\hline 8 & $39 / \mathrm{M}$ & Ependymoma & $\mathrm{C} 4-\mathrm{C} 7$ & MEP change & Transient \\
\hline 9 & $79 / \mathrm{F}$ & Lumbar myelopathy & T9-L2 & MEP change & Transient \\
\hline 10 & $50 / \mathrm{M}$ & LMMC & L4-S3 & Both MEP and SEP change & Transient \\
\hline 11 & $74 / \mathrm{F}$ & Cervical myelopathy & C3-C6 & Both MEP and SEP change & Transient \\
\hline 12 & $41 / \mathrm{M}$ & Spinal stenosis & $\mathrm{T} 2-\mathrm{T} 8$ & Both MEP and SEP change & Transient \\
\hline 13 & $21 / \mathrm{M}$ & Neuromuscular scoliosis & T8-L2 & Both MEP and SEP change & Transient \\
\hline 14 & $29 / \mathrm{F}$ & $\begin{array}{l}\text { Spinal cord tumor, uncertain } \\
\text { type, intramedullary }\end{array}$ & $\mathrm{C} 2-\mathrm{C} 5$ & Both MEP and SEP change & Transient \\
\hline 15 & $41 / \mathrm{F}$ & AS & T6-T10 & MEP change & Transient \\
\hline 16 & $68 / \mathrm{M}$ & Spondylodiscitis & T6-T7 & MEP change & Transient \\
\hline 17 & $44 / \mathrm{F}$ & $\begin{array}{l}\text { Spinal cord tumor, } \\
\text { intramedullary }\end{array}$ & C5-T4 & MEP change & Transient \\
\hline 18 & $52 / \mathrm{F}$ & Cervical disc herniation & C4-C6 & MEP change & Transient \\
\hline 19 & $73 / \mathrm{M}$ & Cervical myelopathy, HCD & $\mathrm{C} 3-\mathrm{C} 7$ & Both MEP and SEP change & Transient \\
\hline 20 & $62 / \mathrm{M}$ & Cervical myelopathy, OPLL & $\mathrm{C} 2-\mathrm{T} 1$ & MEP change & Transient \\
\hline
\end{tabular}

EP, evoked potential; MEP, motor evoked potential; SEP, somatosensory evoked potential; OPLL, ossification of the posterior longitudinal ligament; LMMC, lipomyelomeningocele; AS, ankylosing spondylitis; HCD, herniated cervical disc. 
50 points on each side. Any reduction in motor score (1 point or more) compared with the preoperative state was considered a neurological motor deficit.

Postoperative motor deficits were assessed in all patients. In the group with immediate postoperative motor weakness, a follow-up was conducted 4 weeks later. Deficits that showed improvement in 4 weeks were characterized as transient, and permanent if they persisted after the 4 weeks. Regardless of deficit type, the intraoperative monitoring data were analyzed and the results were confirmed based on the disease classification of each patient.

\section{Statistical analyses}

Data was imported into SPSS ver. 20.1 (IBM, Armonk, NY, USA) for analysis. Based on the characteristics of the variables, we used either an independent-samples t-test or chi-square test to determine significant differences between the preoperative motor intact and motor deficit group in sex, age and diagnoses. Additionally, differences in the rate of postoperative motor deficits between the preoperative status with and without motor impairment were analyzed using chi-square test. Two-sided p-values of $<0.05$ were considered statistically significant.

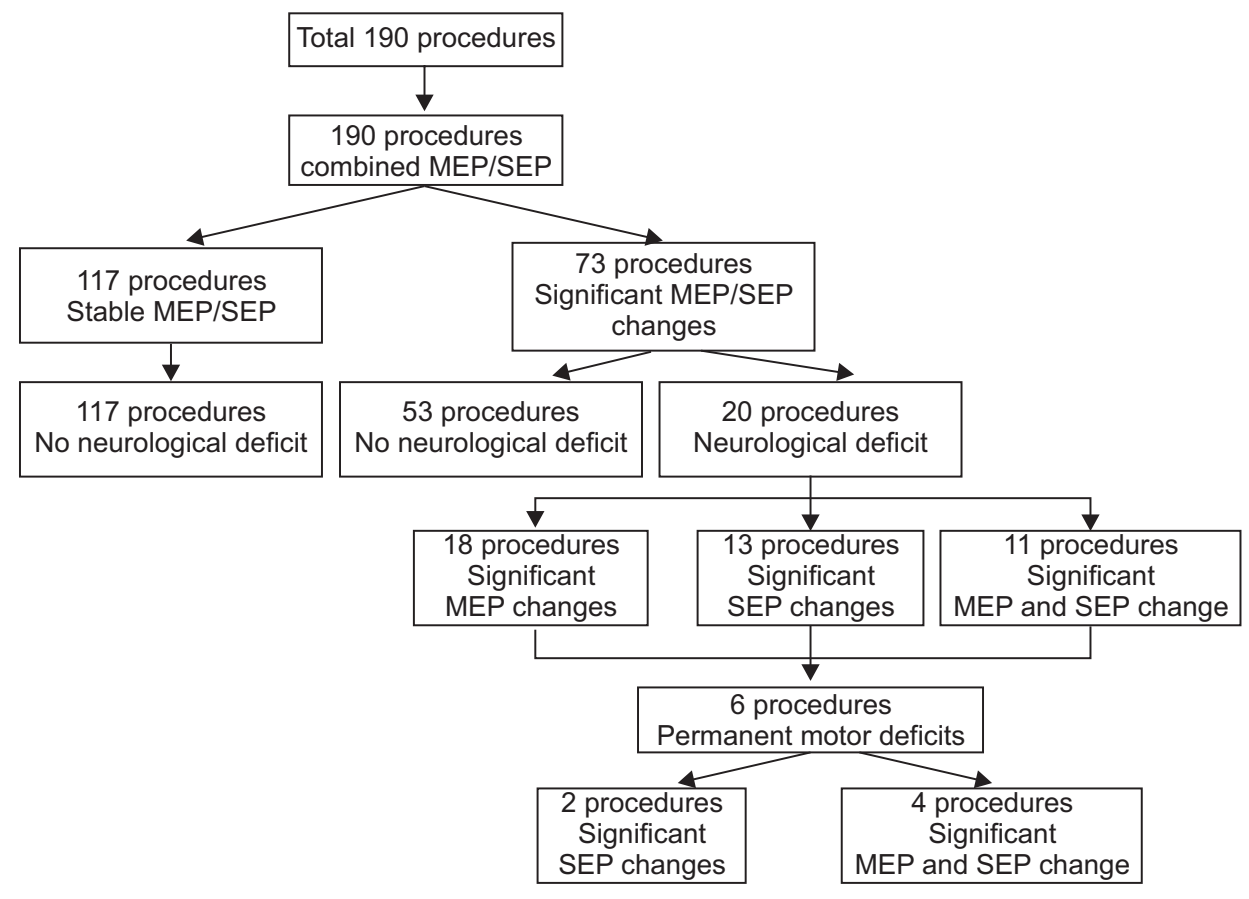

\section{RESULTS}

\section{General characteristics of the subjects}

A total of 190 patients were included in the study; 79 had shown motor deficit preoperatively. Of these, 8 (10.1\%) recovered from their deficits within 48 hours postoperatively. Table 1 compared the group that was neurologically intact preoperatively (group A) and the group with deficits (group B). The mean age was higher in group B than group A, idiopathic scoliosis and syringomyelia were observed more frequently in group $\mathrm{A}$, and neuromuscular scoliosis was observed more frequently in group B. There was no statistically significant difference in age, sex, or eventual diagnoses.

Correlations between intraoperative monitoring and postoperative motor deficits

Stable mMEPs and SEPs were observed in 117 of the 190 patients. Seventy-three patients showed EP changes, and 20 of these 73 showed transient or permanent motor deficits. Of the 20 patients with postoperative motor deficits, 11 patients had changes in both mMEPs and SEPs. mMEP changes alone were observed in 7 patients, and SEP changes alone in 2 patients. Thus, all 20 patients manifested a change in their mMEPs, SEPs, or both (Table 2). Six patients showed motor deficits 4 weeks after having surgery and were assessed for muscle strength. Both
Fig. 1. Intraoperative monitoring and clinical outcome in spinal operations. MEP, motor evoked potential; SEP, somatosensory evoked potential. 
MMEP and SEP changes were observed in 4 of the 6 patients; in the other 2, SEP changes were observed without mMEP changes (Fig. 1).

Correlation between intraoperative monitoring and postoperative motor deficit in each spinal operation

Of the 54 Group 1 patients who had spinal cord tumor operations, 8 showed transient motor deficits, and half transitioned to permanent motor deficits. Of the 4 who showed transient motor deficits, 2 showed mMEP and SEP changes, and the other 2 showed mMEP changes alone. MMEP and SEP changes were observed in 2 of the 4 patients with permanent motor deficits, and the remaining 2 showed SEP changes alone. In Group 2, the 54 patients who had spinal deformity operations, one patient showed transient motor deficits and changes in both mMEPs and SEPs. In Group 3, the 82 patients who had other spinal operations, 11 showed transient motor deficits, 6 of the 11 showed MMEP and SEP changes, and the other 5 showed mMEP changes alone. On a followup conducted 4 weeks postoperatively, 2 patients showed permanent motor deficits as well as MMEP and SEP changes.

\section{Sensitivity and specificity}

Taken altogether, 18 of 20 patients showed transient motor deficits and mMEP changes. Sensitivity was measured at $90 \%$ and mMEP or SEP changes were observed in all 18 patients (100\%). For SEP changes, sensitivity was $65 \%$, but specificity was $85.8 \%$. For combined mMEP and SEP changes, sensitivity was also low (55\%), but, on the other hand, specificity was $95.2 \%$. All 6 patients with combined mMEP and SEP findings showed permanent motor deficits, which resulted in a sensitivity of $100 \%$ in all the patients' SEP changes, and mMEP or SEP changes. For mMEP and SEP changes, specificity indicated a high rate of $91.8 \%$ (Table 3 ).

In all the three groups, the sensitivity of MMEP or SEP changes was $100 \%$. In Group 1 and Group 3, all patients with motor deficits showed mMEP changes (a sensitivity of $100 \%)$. In these groups, the specificity of mMEP and SEP changes was $97.8 \%$ and $92.4 \%$, respectively (Table 4 ).

Table 3. Sensitivity and specificity of intraoperative monitoring in spinal operations

\begin{tabular}{|c|c|c|c|c|}
\hline & \multicolumn{2}{|c|}{ Transient motor deficit } & \multicolumn{2}{|c|}{ Permanent motor deficit } \\
\hline & Sensitivity (\%) & Specificity (\%) & Sensitivity (\%) & Specificity (\%) \\
\hline MEP & 90.0 & 78.2 & 66.6 & 72.2 \\
\hline SEP & 65.0 & 85.8 & 100 & 83.1 \\
\hline Both MEP and SEP & 55.0 & 95.2 & 66.6 & 91.8 \\
\hline Either MEP or SEP & 100 & 68.8 & 100 & 63.5 \\
\hline
\end{tabular}

MEP and SEP changes were defined by this study.

MEP, motor evoked potential; SEP, somatosensory evoked potential.

Table 4. Sensitivity and specificity of intraoperative monitoring with transient motor deficit in each type of spinal operation

\begin{tabular}{lcccccccc}
\hline & \multicolumn{2}{c}{ Group 1 } & & \multicolumn{2}{c}{ Group 2 } & & \multicolumn{2}{c}{ Group 3 } \\
\cline { 2 - 3 } & $\begin{array}{c}\text { Sensitivity } \\
\mathbf{( \% )}\end{array}$ & $\begin{array}{c}\text { Specificity } \\
\mathbf{( \% )}\end{array}$ & & $\begin{array}{c}\text { Sensitivity } \\
\mathbf{( \% )}\end{array}$ & $\begin{array}{c}\text { Specificity } \\
\mathbf{( \% )}\end{array}$ & $\begin{array}{c}\text { Sensitivity } \\
\mathbf{( \% )}\end{array}$ & $\begin{array}{c}\text { Specificity } \\
\mathbf{( \% )}\end{array}$ \\
\hline MEP & 75.0 & 84.7 & & 100 & 64.1 & 100 & 84.5 \\
SEP & 75.0 & 95.6 & & 100 & 84.9 & & 54.5 & 78.8 \\
Both MEP and SEP & 50.0 & 97.8 & & 100 & 92.4 & & 54.5 & 94.3 \\
Either MEP or SEP & 100 & 73.9 & & 100 & 56.6 & & 100 & 69.0 \\
\hline
\end{tabular}

MEP and SEP changes were defined by this study.

Group 1, spinal cord tumor operations; Group 2, spinal deformity operations; Group 3, other spinal operations; MEP, motor evoked potential; SEP, somatosensory evoked potential. 
Table 5. Comparison between preoperative motor intact group and motor deficit group in postoperative motor outcome (group A, preoperatively motor intact; group B, preoperatively motor deficit)

\begin{tabular}{cccc}
\hline $\begin{array}{c}\text { Postoperative } \\
\text { motor outcome }\end{array}$ & $\begin{array}{c}\text { Group A } \\
(\mathbf{n = 1 1 1 )}\end{array}$ & $\begin{array}{c}\text { Group B } \\
(\mathbf{n = 7 9 )}\end{array}$ & p-value \\
\hline No deficit & $104(93.7)$ & $66(83.5)$ & 0.031 \\
\hline Deficit & $7(6.3)$ & $13(16.5)$ & \\
\hline
\end{tabular}

Values are presented as number (\%).

Comparison between group with preoperative motor intact and deficit

Thirteen (16.5\%) of the 79 patients with preoperative motor deficits also had postoperative motor deficits, whereas 7 (6.3\%) of the 111 patients who were motor intact preoperatively developed postoperative motor deficits. The prevalence of postoperative motor deficits was significantly higher $(\mathrm{p}<0.01)$ in the preoperative motor deficit group (Table 5).

\section{DISCUSSION}

Combined mMEP and SEP monitoring provides a great deal of information for spinal cord surgery. In this study, we monitored 190 patients in all, and could observe EP changes in 73 and neurological weakness in 20. In 18 of the 20 patients, the sensitivities of mMEP changes and mMEP or SEP changes were measured at $90 \%$ and $100 \%$, respectively. For mMEP and SEP changes, specificity was $95.2 \%$. In all the patients who showed motor deficits subsequent to spinal deformity surgery and other spinal surgery, mMEP changes were observed. Likewise, changes during each surgery also showed high specificity. In 6 patients, permanent neurological deficits remained, 4 of who had been treated for spinal cord tumors. There were no complaints of headache, seizures, or skin burns postoperatively. The most common concern was direct cortical thermal injury; but over the last 15 years only two cases have been reported [6]. In a 2002 survey of the literature, published complications included tongue laceration, cardiac arrhythmia, scalp burning at the site of the stimulating electrodes, jaw fracture and awareness [7].

Although SEP monitoring may be a reliable way to guide spinal surgery, many studies have reported that it is inappropriate to evaluate motor pathway functional integrity, and that it shows high false-negative rates [8-10].
Kundnani et al. [8] performed combined monitoring on 354 patients undergoing idiopathic scoliosis operations. SEP monitoring in that study showed a specificity of $100 \%$ but a sensitivity of $51 \%$. Deutsch et al. [9] performed SEP monitoring on 44 cases of anterior thoracic vertebrectomy, and showed a high false-negative rate and a sensitivity of $0 \%$. Hilibrand et al. [10] reported that in cervical operations, SEPs showed a sensitivity of $25 \%$ and a specificity of $100 \%$.

Many previous studies have reported that the inability of SEP monitoring to detect motor symptoms and its associated false negatives are because it covers the dorsal sensory tract rather than the ventral motor tract [11]. Moreover, SEPs are less sensitive in detecting nerve root injuries and thus could miss injuries caused by the process of pedicle screw placement or nerve root traction [12]. Such problems can be limits of SEPs' use for a standalone monitoring tool. Nonetheless, in this study, SEP monitoring showed high sensitivity and specificity in patients with permanent motor deficits. Garcia et al. [13] conducted SEP monitoring in 80 patients who had undergone cervical laminoplasty, with sensitivity and specificity results of $100 \%$ and $99 \%$, respectively, which implies that SEPs can useful for confirming postoperative motor deficits.

Based on the site of stimulation or recording, intraoperative electrical MEPs can be further classified. For example, MEPs can be recorded over muscle (mMEPs) or over the peripheral nerves (neurogenic MEPs). Some previous studies $[14,15]$ have reported that neurogenic MEPs primarily track sensory, rather than motor, responses and are mediated by the same neural pathways as SEPs. As such, current consensus is against the use of neurogenic MEPs as the sole method of motor pathway monitoring $[16,17]$. However, mMEPs are the more frequently reported modality of MEP monitoring because of the relative simplicity in generating and recording MEPs. mMEPs are of higher sensitivity than SEPs and can detect spinal motor damage directly. However, Modi et al. [18] reported that spinal damage might occur due to blood loss during an operation, and thus that MMEP monitoring is inadequate for the sensitive detection of ischemic cord injury. Thus, the two modalities provide complementary information in the process of postoperative monitoring. In this study, we conducted multimodal monitoring by considering 4 cases: SEPs alone, mMEPs alone, mMEPs 
and SEPs together, and either SEPs or mMEPs alone and analyzed the results.

mMEP or SEP changes were observed in all 20 patients with neurological deficits, and both MMEP and SEP changes were observed in 11 of these patients. mMEP changes alone were observed in 7 patients, and SEP changes alone were observed in 2 patients. In predicting immediate postoperative neurological deficits, mMEP sensitivity was $90 \%$, and sensitivity of either MMEP or SEP change was $100 \%$. Overall SEP sensitivity stood at $65 \%$. Combined mMEPs and SEPs showed sensitivity of $55 \%$. With respect to neurological deficits evaluated after 4 weeks, SEP sensitivity was $100 \%$, but combined mMEPs and SEPs, as well as mMEPs alone, had a sensitivity of only $66.6 \%$. These results might be explained by SEP monitoring showing a low sensitivity early in the process of immediate postoperative monitoring.

This study analyzed sensitivity in general, but also after specific surgeries, e.g., spinal cord tumor surgery, spinal deformity surgery, and other spinal surgery. In the spinal deformity surgery, combined mMEP and SEP monitoring showed a sensitivity of $100 \%$ and a specificity of $92.4 \%$, values that were higher overall than previous reports $[8,19,20]$. However, for spinal cord tumor surgery, SEP sensitivity and mMEP sensitivity showed low values overall. For other spinal surgery, SEP monitoring showed low sensitivity, which had the effect of lowering combined mMEP and SEP sensitivity, and also lowered the sensitivity of monitoring in spinal surgery overall. In regard to the especially low sensitivity found in spinal cord tumor surgery, recent studies have reported that initial postoperative deterioration ranged from $18 \%-24.6 \%$ $[21,22]$. However, Sala et al. [22] represented that a shorttime evaluation was not enough to compare monitored and non-monitored groups, and that follow-ups should be conducted for at least 3 months to observe whether monitoring produces differences in neurological status. Additionally, it may be difficult to induce mMEPs from patients in whom large tumors have caused spinal cord compression or who have had operations or radiation therapies [23]. In addition, postoperative neurological status is likely to be affected by the surgeon performing the operation, the surgical approach they take, and other underlying diseases not reflected intraoperatively, which might influence the sensitivity of monitoring in other spinal surgery.
Comparison of motor outcomes between the preoperatively intact and deficient motor groups showed significant differences in the rate of postoperative motor deficits, which were higher in patients who had preoperative motor deficits in this study. This may be explained by the vulnerable status of individuals who already had compressed spinal cords amongst the preoperative motor deficit cases. Of the 20 patients in whom neurological deficits occurred, 14 patients showed motor recovery by 4 weeks postoperatively. Thus, such a transient change in muscular strength, namely muscular weakness, was produced by either reversible damage or postoperative pain. In the other 6 patients, their neurological deficits persisted; 4 of these patients showed MMEP and SEP changes, and 2 showed SEP changes alone. As a result, SEP monitoring sensitivity reached $100 \%$ for permanent motor deficits related to spinal surgery, and mMEP monitoring sensitivity was $66 \%$. Most of the patients who showed permanent motor deficits had spinal cord tumors. The other 2 patients underwent OPLL surgery. The 4 patients who had spinal tumor surgery showed mMEP and SEP changes, but the 2 patients with intramedullary cervical cord tumors showed SEP changes alone. The surgery for intramedullary tumors involves a myelotomy, which may lead to the loss of SEPs [2], but mMEPs are frequently unaffected by the loss of SEPs. One patient undergoing this surgery showed a mild ( 2 point) neurological deficit, which did not change at follow-up. A mMEP change might not be expected with such a mild change. Additionally, it is likely that the pathology and progression of a tumor itself can affect motor deficits on long-term follow-up. The two patients who showed SEP changes alone had glioblastoma and anaplastic astrocytoma. Collectively, our results demonstrated that SEPs and mMEPs have complementary roles in evaluating motor deficits and should be considered together rather than alone.

This study used amplitude variation as a criterion of mMEP change when the baseline decreased by $50 \%$ or more $[4,24-26]$. Thus, if the baseline was $80 \%$, it might miss 5 patients who showed permanent neurological deficits, and also increase the false negative rate. Some studies have reported high sensitivities or specificities despite a baseline change of $50 \%$ [11,27], but contrarily, setting the baseline at $50 \%$ for surgery on 29 patients with cervical kyphosis resulted in mMEPs with a sensitivity of $75 \%$ and a specificity of $84 \%$ [25]. In 1,445 cases of anterior 
cervical surgery with a baseline of $60 \%$, mMEP changes were observed in 267 patients, but neurological deficits were observed in only 2 [26]. On the whole, a tendency towards excessive false-alarms appears and specificity falls when the cutoff is set between $50 \%-60 \%$.

In our study, propofol and remifentanil were the primary anesthetics used; desflurane was given as a supplement in only 7 surgeries. mMEP monitoring is more affected by inhalation anesthesia and muscle relaxants, as compared to neurogenic MEPs [28,29]. mMEPs are especially sensitive to the effects of halogenated anesthetics that depress mMEP responses by depressing anterior horn neuron excitability [30]. This suppression by inhalation agents is dose dependent, has the effect of MEP amplitude reduction [31], and results in the wide advocation of intravenous over inhalation anesthetics during mMEP monitoring. However, in our 7 operations with added desflurane, no depression in mMEP monitoring was seen.

While some previous studies have analyzed monitoring efficacy subdivided by surgery type, this was the first to analyze sensitivity and specificity in various spinal cord diseases and to also conduct follow-ups on postoperative neurological deficits and later motor recovery. Nonetheless, it has its limitations. The first is that different surgeons performed their operations in different ways and the patients' underlying diseases or conditions were not reflected in the study. Moreover, this study strictly defined 'motor weakness' as even a single point fall on the MRC scale. Therefore, it included even the most mild weakness, which presumably also affected sensitivity and specificity. An additional weakness of our study is that sensory deficits were not checked and we only focused on the motor outcomes. The follow-up, conducted 1 month later, is also a limitation. Additional follow-ups at 6 and 12 months would enable better interpretations of the benefits of SEP and mMEP monitoring. Additionally, this study focused on SEP and mMEP monitoring, but a combination of mMEP and D-wave recording is usually regarded as an optimal way to evaluate the motor pathway in spinal cord surgery [22], and the application of the absence-presence ground and the epidural D-wave reduces the false positive rate [2]. In addition, free EMG can be used to monitor the motor tract during spinal cord surgery [32]. Multimodal monitoring provides surgeons with more complete neurological information, which is expected to provide additional information regarding risk factors, and further enhance sensitivity.

Intraoperative monitoring is often used during spinal surgery. From our data, we can draw conclusions as follow: the sensitivity of SEP monitoring was $100 \%$ in the case of spinal deformity surgery. Also, in the case of spinal tumor surgery, SEP monitoring enhanced sensitivity and specificity when used with MEP monitoring. For that reason, the use of SEP can be considerable a good option for spinal cord monitoring and combined SEP and mMEP monitoring should be conducted simultaneously in order to prevent further neurological injury.

In the future, additional kinds of intraoperative monitoring and longer follow-up periods should be conducted in order to better predict postoperative motor weakness.

\section{CONFLICT OF INTEREST}

No potential conflict of interest relevant to this article was reported.

\section{ACKNOWLEDGMENTS}

This study was supported by the Je Won Research Foundation (No. 2013-31-0873).

\section{REFERENCES}

1. Szelenyi A, Langer D, Kothbauer K, De Camargo AB, Flamm ES, Deletis V. Monitoring of muscle motor evoked potentials during cerebral aneurysm surgery: intraoperative changes and postoperative outcome. J Neurosurg 2006;105:675-81.

2. Kothbauer K, Deletis V, Epstein FJ. Intraoperative spinal cord monitoring for intramedullary surgery: an essential adjunct. Pediatr Neurosurg 1997;26:247-54.

3. Sala F, Bricolo A, Faccioli F, Lanteri P, Gerosa M. Surgery for intramedullary spinal cord tumors: the role of intraoperative (neurophysiological) monitoring. Eur Spine J 2007;16 Suppl 2:S130-9.

4. Pelosi L, Lamb J, Grevitt M, Mehdian SM, Webb JK, Blumhardt LD. Combined monitoring of motor and somatosensory evoked potentials in orthopaedic spinal surgery. Clin Neurophysiol 2002;113:1082-91.

5. Choi I, Hyun SJ, Kang JK, Rhim SC. Combined muscle motor and somatosensory evoked potentials for intra- 
medullary spinal cord tumour surgery. Yonsei Med J 2014;55:1063-71.

6. Macdonald DB. Intraoperative motor evoked potential monitoring: overview and update. J Clin Monit Comput 2006;20:347-77.

7. Legatt AD. Current practice of motor evoked potential monitoring: results of a survey. J Clin Neurophysiol 2002;19:454-60.

8. Kundnani VK, Zhu L, Tak H, Wong H. Multimodal intraoperative neuromonitoring in corrective surgery for adolescent idiopathic scoliosis: evaluation of 354 consecutive cases. Indian J Orthop 2010;44:64-72.

9. Deutsch H, Arginteanu M, Manhart K, Perin N, Camins M, Moore F, et al. Somatosensory evoked potential monitoring in anterior thoracic vertebrectomy. J Neurosurg 2000;92(2 Suppl):155-61.

10. Hilibrand AS, Schwartz DM, Sethuraman V, Vaccaro AR, Albert TJ. Comparison of transcranial electric motor and somatosensory evoked potential monitoring during cervical spine surgery. J Bone Joint Surg Am 2004;86-A:1248-53.

11. Weinzierl MR, Reinacher P, Gilsbach JM, Rohde V. Combined motor and somatosensory evoked potentials for intraoperative monitoring: intra- and postoperative data in a series of 69 operations. Neurosurg Rev 2007;30:109-16.

12. Lall RR, Lall RR, Hauptman JS, Munoz C, Cybulski GR, Koski T, et al. Intraoperative neurophysiological monitoring in spine surgery: indications, efficacy, and role of the preoperative checklist. Neurosurg Focus 2012;33:E10.

13. Garcia RM, Qureshi SA, Cassinelli EH, Biro CL, Furey CG, Bohlman HH. Detection of postoperative neurologic deficits using somatosensory-evoked potentials alone during posterior cervical laminoplasty. Spine J 2010;10:890-5.

14. Toleikis JR, Skelly JP, Carlvin AO, Burkus JK. Spinally elicited peripheral nerve responses are sensory rather than motor. Clin Neurophysiol 2000;111:736-42.

15. Minahan RE, Sepkuty JP, Lesser RP, Sponseller PD, Kostuik JP. Anterior spinal cord injury with preserved neurogenic 'motor' evoked potentials. Clin Neurophysiol 2001;112:1442-50.

16. Deletis V, Sala F. Intraoperative neurophysiological monitoring of the spinal cord during spinal cord and spine surgery: a review focus on the corticospinal tracts. Clin Neurophysiol 2008;119:248-64.

17. Malhotra NR, Shaffrey CI. Intraoperative electrophysiological monitoring in spine surgery. Spine (Phila Pa 1976) 2010;35:2167-79.

18. Modi HN, Suh SW, Yang JH, Yoon JY. False-negative transcranial motor-evoked potentials during scoliosis surgery causing paralysis: a case report with literature review. Spine (Phila Pa 1976) 2009;34:E896-900.

19. Bhagat S, Durst A, Grover H, Blake J, Lutchman L, Rai AS, et al. An evaluation of multimodal spinal cord monitoring in scoliosis surgery: a single centre experience of 354 operations. Eur Spine J 2015;24:1399-407.

20. Zhuang Q, Wang S, Zhang J, Zhao H, Wang Y, Tian Y, et al. How to make the best use of intraoperative motor evoked potential monitoring? Experience in 1162 consecutive spinal deformity surgical procedures. Spine (Phila Pa 1976) 2014;39:E1425-32.

21. Garces-Ambrossi GL, McGirt MJ, Mehta VA, Sciubba DM, Witham TF, Bydon A, et al. Factors associated with progression-free survival and long-term neurological outcome after resection of intramedullary spinal cord tumors: analysis of 101 consecutive cases. J Neurosurg Spine 2009;11:591-9.

22. Sala F, Palandri G, Basso E, Lanteri P, Deletis V, Faccioli $\mathrm{F}$, et al. Motor evoked potential monitoring improves outcome after surgery for intramedullary spinal cord tumors: a historical control study. Neurosurgery 2006;58:1129-43.

23. Hsu W, Bettegowda C, Jallo GI. Intramedullary spinal cord tumor surgery: can we do it without intraoperative neurophysiological monitoring? Childs Nerv Syst 2010;26:241-5.

24. Langeloo DD, Lelivelt A, Louis Journee H, Slappendel $\mathrm{R}$, de Kleuver M. Transcranial electrical motor-evoked potential monitoring during surgery for spinal deformity: a study of 145 patients. Spine (Phila Pa 1976) 2003;28:1043-50.

25. Park P, Wang AC, Sangala JR, Kim SM, Hervey-Jumper $\mathrm{S}$, Than KD, et al. Impact of multimodal intraoperative monitoring during correction of symptomatic cervical or cervicothoracic kyphosis. J Neurosurg Spine 2011;14:99-105.

26. Lee JY, Hilibrand AS, Lim MR, Zavatsky J, Zeiller S, Schwartz DM, et al. Characterization of neurophysiologic alerts during anterior cervical spine surgery. Spine (Phila Pa 1976) 2006;31:1916-22. 
27. Sutter M, Eggspuehler A, Grob D, Jeszenszky D, Benini A, Porchet F, et al. The validity of multimodal intraoperative monitoring (MIOM) in surgery of 109 spine and spinal cord tumors. Eur Spine J 2007;16 Suppl 2:S197-208.

28. Haghighi SS, Madsen R, Green KD, Oro JJ, Kracke GR. Suppression of motor evoked potentials by inhalation anesthetics. J Neurosurg Anesthesiol 1990;2:73-8.

29. Chen Z. The effects of isoflurane and propofol on intraoperative neurophysiological monitoring during spinal surgery. J Clin Monit Comput 2004;18:303-8.

30. Lotto ML, Banoub M, Schubert A. Effects of anes- thetic agents and physiologic changes on intraoperative motor evoked potentials. J Neurosurg Anesthesiol 2004;16:32-42.

31. Huotari AM, Koskinen M, Suominen K, Alahuhta S, Remes R, Hartikainen KM, et al. Evoked EEG patterns during burst suppression with propofol. Br J Anaesth 2004;92:18-24.

32. Skinner SA, Nagib M, Bergman TA, Maxwell RE, Msangi G. The initial use of free-running electromyography to detect early motor tract injury during resection of intramedullary spinal cord lesions. Neurosurgery 2005;56(2 Suppl):299-314. 